\title{
COMMUTATOR IDEALS AND SEMICOMMUTATOR IDEALS OF TOEPLITZ ALGEBRAS ASSOCIATED WITH FLOWS
}

\author{
PAUL S. MUHLY AND JINGBO XIA
}

(Communicated by Palle E. T. Jorgensen)

\begin{abstract}
We prove that for a Toeplitz $C^{*}$-algebra associated with a flow that has no fixed points, the commutator ideal and the semicommutator ideal coincide.
\end{abstract}

\section{INTRODUCTION}

This is a continuation of our study of Toeplitz algebras associated with flows $[2,6,8,9]$. Consider the $C^{*}$-algebra $\mathscr{T}(C)$ generated by Toeplitz operators $\left\{T_{\varphi}: \varphi \in C\right\}$ on $H^{2}(\mathbb{R})$, where $C$ is a $C^{*}$-algebra of bounded, uniformly continuous functions on $\mathbb{R}$ that is closed under the natural translation. This algebra has been studied in various contexts. Two of the most important aspects concerning $\mathscr{T}(C)$ are its ideal structure and generalized Fredholm theory for operators in $\mathscr{T}(C)$. In either case, one inevitably encounters the ideal $\mathscr{C}_{1 / 2}(C)$ of $\mathscr{T}(C)$ generated by semicommutators $\left\{T_{\varphi} T_{\psi}-T_{\varphi \psi}: \varphi, \psi \in C\right\}$. As it turns out, the quotient algebra $\mathscr{T}(C) / \mathscr{C}_{1 / 2}(C)$ is always isomorphic to $C$ itself. After the identification of this quotient algebra, one can proceed to establish an index theory that identifies topological invariants of $C$ with analytic invariants of a von Neumann algebra into which $\mathscr{T}(C)$ is embedded [2, 3]. All this is very nice until one develops the uneasy feeling that there might be something unnatural involved here. The source of this uneasiness is the semicommutator ideal $\mathscr{C}_{1 / 2}(C)$. As it is defined, $\mathscr{C}_{1 / 2}(C)$ is not a natural algebraic object; its definition depends on properties that only Toeplitz operators possess. One cannot help feeling that $\mathscr{C}_{1 / 2}(C)$ is too much of an ad-hoc concoction. In contrast, the ideal $\mathscr{C}(C)$ of $\mathscr{T}(C)$ generated by commutators seems to be a much more natural algebraic object. After all, every $C^{*}$-algebra has a commutator ideal, and the quotient is, of course, a commutative $C^{*}$-algebra. Because of the naturalness of $\mathscr{C}(C)$, it is more desirable to establish an index theory for $\mathscr{T}(C)$ corresponding to the exact sequence

$$
0 \rightarrow \mathscr{C}(C) \rightarrow \mathscr{T}(C) \rightarrow \mathscr{T}(C) / \mathscr{C}(C) \rightarrow 0 .
$$

However, the trouble with this possible index theory is that, except for the few

Received by the editors April 20, 1991.

1991 Mathematics Subject Classification. Primary 47B35, 47B47, 47C15.

Supported in part by grants from the National Science Foundation.

(C) 1992 American Mathematical Society $0002-9939 / 92 \$ 1.00+\$ .25$ per page 
cases where $\mathscr{C}(C)$ and $\mathscr{C}_{1 / 2}(C)$ are known to coincide, the quotient algebra $\mathscr{T}(C) / \mathscr{C}(C)$ is not calculable for an arbitrary flow.

So the situation we face is that, on the one hand, we can establish a nice index theory for $\mathscr{T}(C)$ with an apparently unnatural ideal while, on the other hand, we have a perfectly natural ideal that has little use in the study of the Toeplitz algebra. This calls for a thorough investigation of the relation between the two ideals. The most tempting thought is that it would be nice if these ideals turned out to be the same. But examples tell us that this is not the case. However, it is quite easy to show that if the flow induced by the natural translation on $C$ is uniquely ergodic, then the two ideals are the same [2]. But even at the time when [2] was being written, we had the feeling that the $\mathscr{C}_{1 / 2}(C)$ and $\mathscr{C}(C)$ should coincide for a much larger class of flows. This class, we felt, should include, at the very least, every minimal flow. The purpose of this note is to show that for any flow without fixed points, these ideals coincide.

\section{THE MAIN RESULT}

Let $X$ be a compact Hausdorff space upon which $\mathbb{R}$ acts as a group of homeomorphisms. Write $x+t$ for the translation of $x \in X$ by $t \in \mathbb{R}$. Assume that $(x, t) \mapsto x+t$ is a continuous map from $X \times \mathbb{R}$ to $X$. We refer to the action of $\mathbb{R}$ on $X$ as a flow. Let $A(X)$ denote the collection of continuous functions $\varphi$ on $X$ such that for every $x \in X$, the function $t \mapsto \varphi(x+t)$ belongs to $H^{\infty}(\mathbb{R})$.

Definition. An analytic representation of the flow $(X, \mathbb{R})$ is a pair $(\pi, P)$ where $\pi$ is a $C^{*}$-algebra representation of $C(X)$ on some Hilbert space $K$ and $P$ is an orthogonal projection on $K$ satisfying

(1.1) $\pi(1)=1$,

(1.2) $\pi(\varphi) P=P \pi(\varphi) P$ for every $\varphi \in A(X)$.

We say that an analytic representation $(\pi, P)$ of the flow is faithful if the $C^{*}$ algebra $\mathscr{A}$ generated by $\pi(C(X))$ and $P$ is noncommutative.

The reason for the term "faithful" is that in [9] it is shown that there exists a universal $C^{*}$-algebra with the property that there is a bijective correspondence between $C^{*}$-representations of it and analytic representations of the flow; and an analytic representation gives rise to a faithful $C^{*}$-representation if and only if it is faithful in the sense just defined.

Let an analytic representation $(\pi, P)$ of the flow be given. Let $\mathscr{T}$ denote the Toeplitz algebra associated with $(\pi, P)$. That is, $\mathscr{T}=P \mathscr{A} P$. $\mathscr{T}$ is regarded both as a subalgebra of $\mathscr{A}$ and as a unital $C^{*}$-algebra on the subspace $P K$. Let $\mathscr{C}$ denote the ideal in $\mathscr{T}$ generated by the commutators $\{A B-B A: A, B \in$ $\mathscr{T}\} . \mathscr{C}$ is the same as the ideal of $\mathscr{T}$ generated by

$$
\{P \pi(\varphi) P \pi(\psi) P-P \pi(\psi) P \pi(\varphi) P: \varphi, \psi \in C(X)\} .
$$

Let $\mathscr{C}_{1 / 2}$ denote the ideal of $\mathscr{T}$ generated by the semicommutators

$$
\{P \pi(\varphi) P \pi(\psi) P-P \pi(\varphi \psi) P: \varphi, \psi \in C(X)\} .
$$

Obviously $\mathscr{C}_{1 / 2} \supset \mathscr{C}$. Despite having similar appearances, $\mathscr{C}_{1 / 2}$ and $\mathscr{C}$ do not always coincide. We first present an example where they differ. This example derives from a well-known result of Gohberg and Krupnik [5], but in their paper, 
the setting is quite different. They do not regard their Toeplitz algebras as one associated with a flow but, rather, as one associated with the unit disc.

Example. Consider $C_{*, *}(\mathbb{R})$, the collection of continuous functions $f$ on $\mathbb{R}$ such that the limits $\lim _{s \rightarrow+\infty} f(s)$ and $\lim _{s \rightarrow-\infty} f(s)$ exist but are not necessarily equal. Let $X$ be the maximal ideal space of $C_{*, *}(\mathbb{R})$. It is elementary that $X$ is the two-point compactification $\{-\infty\} \cup \mathbb{R} \cup\{+\infty\}$ of $\mathbb{R}$. The natural translation of functions induces a flow $\left\{\alpha_{t}: t \in \mathbb{R}\right\}$ on $X$ such that for $x$ in the subset $\mathbb{R}$ of $X=\{-\infty\} \cup \mathbb{R} \cup\{+\infty\}, \alpha_{t}(x)=x+t$ and $\alpha_{t}$ fixes both $-\infty$ and $+\infty$. Let $K=L^{2}(\mathbb{R})$ and let $P$ be the projection from $L^{2}(\mathbb{R})$ onto $H^{2}(\mathbb{R})$. Further, let $\pi: C(X) \rightarrow \mathscr{L}\left(L^{2}(\mathbb{R})\right)$ be the representation

$$
\pi(\hat{f}) g=P(f g), \quad g \in L^{2}(\mathbb{R}),
$$

for every $f \in C_{*, *}(\mathbb{R})$. ( $\hat{f}$ is the Gelfand transform of $\left.f.\right)$ This pair $(\pi, P)$ is a nondegenerate analytic representation of the flow. By the results of [5], for this pair of $(\pi, P)$, we have $\mathscr{C}_{1 / 2} / \mathscr{C} \cong C_{0}(0,1)$. As we will see from our main result, the fact that this flow has fixed points is the key here.

It was shown in $[2$, Lemma 24.1$]$ that if the flow $(X, \mathbb{R})$ is uniquely ergodic, meaning that the flow possesses only one invariant probability measure, then $\mathscr{C}_{1 / 2}=\mathscr{C}$. The main purpose of this paper is to show that the equality $\mathscr{C}_{1 / 2}=\mathscr{C}$ holds for a much larger class of flows.

Theorem 1. If the flow $(X, \mathbb{R})$ has no fixed points then $\mathscr{C}_{1 / 2}=\mathscr{C}$.

Let $C_{\mathrm{bu}}(\mathbb{R})$ denote the collection of bounded, uniformly continuous functions on $\mathbb{R}$. For any subset $F \subset C_{\mathrm{bu}}(\mathbb{R})$, let $C_{F}$ denote the unital $C^{*}$-algebra generated by $F$ together with all its translations on $\mathbb{R}$. Let $X_{F}$ be the maximal ideal space of $C_{F}$. Let $\left(X_{F}, \mathbb{R}\right)$ be the flow on $X_{F}$ induced by the natural translation of functions in $C_{F}$. This flow is sometimes called the Beboutov flow associated with the family $F$. It is quite easy to derive a necessary condition for the existence of fixed points for this flow. The following lemma is completely elementary and so we will not present its proof.

Lemma 1.1. Suppose that the flow $\left(X_{F}, \mathbb{R}\right)$ has at least one fixed point. Then there exist sequences $\left\{t_{n}\right\} \subset \mathbb{R}$ and $\left\{T_{n}\right\} \subset(0, \infty)$ such that

$$
\begin{aligned}
& \lim _{n \rightarrow \infty}\left|t_{n}\right|=\infty, \\
& \lim _{n \rightarrow \infty} T_{n}=\infty, \text { and } \\
& \lim _{n \rightarrow \infty} \sup \left\{|g(s)-g(t)|: s, t \in\left[t_{n}-T_{n}, t_{n}+T_{n}\right]\right\}=0
\end{aligned}
$$

for every $g \in F$.

Consequently, if $F$ contains a function $h$ with the property that there exist $c>0$ and $r>0$ such that for any $t \in \mathbb{R}, h([t-c, t+c])$ is not contained in any disc of radius $r$, then the flow $\left(X_{F}, \mathbb{R}\right)$ has no fixed points. There are many elementary functions that are uniformly continuous and have this property. For example, with $a \neq 0$,

$$
h(t)=\sin (a t+b)
$$

has this property as do each of the following functions:

$$
\begin{aligned}
& h(t)=\sin (a t+b \log (1+|t|)+c), \\
& h(t)=\sin \left(a t+b_{1} \log (1+|t|)+b_{2}|t|^{1 / 2}+b_{3} \log (\log (1+|t|))\right), \\
& h(t)=\cos \left(a t+b \sin \left(\log \left(1+|t|^{4 / 5}\right)\right)\right) .
\end{aligned}
$$


If $F$ contains any of these functions, then by Theorem 1, the commutator ideal and the semicommutator ideal of the usual Toeplitz operators $\left\{T_{\varphi}: \varphi \in C_{F}\right\}$ on $H^{2}(\mathbb{R})$ are the same.

The example we gave earlier shows that if the flow has at least two fixed points, then $\mathscr{C}_{1 / 2}$ and $\mathscr{C}$ may differ. At this point it is natural to ask what happens when the flow has only one fixed point? Consider the natural flow on the one point compactification of $\mathbb{R}$, i.e., the flow that is the natural translation on $\mathbb{R}$ and fixes the infinity. For this flow, $\mathscr{C}_{1 / 2}$ and $\mathscr{C}$ are the same. But does there exist a flow with a single fixed point for which $\mathscr{C}_{1 / 2}$ and $\mathscr{C}$ differ? Or is it true that $\mathscr{C}_{1 / 2}$ and $\mathscr{C}$ always coincide if the flow has no more than one fixed point? Another question is: Must $\mathscr{C}_{1 / 2}$ and $\mathscr{C}$ differ if the flow has two or more fixed points and the space is connected? We do not know the answers to these questions.

The rest of the paper is devoted to the proof of Theorem 1 . Obviously both $\mathscr{C}_{1 / 2}$ and $\mathscr{C}$ may be reduced to $\{0\}$ if the representation $(\pi, P)$ fails to be faithful. So for the rest of the paper, $(\pi, P)$ is assumed to be faithful. The proof requires some technical preparation, which we will take care of first.

\section{SPECTRUM AND FIXED POINTS}

For each function $\varphi \in C(X)$, let $\operatorname{sp}(\varphi)$ denote the spectrum, in the sense of spectral synthesis, of the function with respect to the flow $(X, \mathbb{R})$. (Readers not familiar with spectral synthesis are referred to $[1,4,7,10]$ for detailed discussions of the subject.) The following facts are well known:

(2.1) $A(X)=\{\varphi: \varphi \in C(X), \operatorname{sp}(\varphi) \subset[0, \infty)\}$;

(2.2) $\operatorname{sp}(\bar{\varphi})=-\operatorname{sp}(\varphi)$;

(2.3) $\operatorname{sp}(\varphi \psi) \subset[a+b, \infty)$ if $\operatorname{sp}(\varphi) \subset[a, \infty)$ and $\operatorname{sp}(\psi) \subset[b, \infty)$;

(2.4) $\{\varphi: \varphi \in C(X), \operatorname{sp}(\varphi)$ is bounded set $\}$ is dense in $C(X)$.

For a set $S \subset \mathbb{R}$, let

$$
C(X, S)=\{\varphi: \varphi \in C(X), \operatorname{sp}(\varphi) \subset S\} .
$$

Let $d m$ be an invariant probability measure on $X$. The flow on $X$ induces a group of unitary $\left\{u_{t}: t \in \mathbb{R}\right\}$ on $L^{2}(X, d m)$; that is, $\left(u_{t} g\right)(x)=g(x+t)$ for every $g \in L^{2}(X, d m)$. Write $u_{t}=\exp (i t \delta)$, where $\delta$ is the infinitesimal generator of the unitary group. The operator $\delta$ has the spectral decomposition

$$
\delta=\int_{\mathbb{R}} \lambda d e_{\lambda}
$$

on $L^{2}(X, d m)$. For any open interval $I \subset \mathbb{R}, C(X, S) \subset e(I) L^{2}(X, d m)$ whenever the closure of $S$ is contained in $I$. Furthermore,

$$
e(I) L^{2}(X, d m)=\bigvee\{C(X, S): \bar{S} \subset I\} .
$$

Here, the symbol $\vee$ means "the closure of the linear span of".

Proposition 2.1. The following are equivalent:

(a) The flow $(X, \mathbb{R})$ has no fixed points.

(b) For any invariant probability measure $d m$ of the flow $(X, \mathbb{R})$, the spectrum of the infinitesimal generator $\delta$ of $\left\{u_{t}: t \in \mathbb{R}\right\}$ on $L^{2}(X, d m)$ contains $c \mathbb{Z}$ with some $c>0$. 
Proof. (a) $\Rightarrow$ (b) By the spectral analysis of the flow, if $c \in \sigma(\delta)$ then $\sigma(\delta) \supset$ $c \mathbb{Z}$. Therefore it suffices to rule out the possibility that $\sigma(\delta)=\{0\}$. If it were the case that $\sigma(\delta)=\{0\}$, then every $u_{t}$ is the identity operator. Therefore, for every $\varphi \in C(X), \varphi=u_{t} \varphi=\varphi$ as elements in $L^{2}(X, d m)$. Since $\varphi$ is continuous, $\varphi(x+t)=\varphi(x)$ for every $x$ in the (closed) support of $d m$; and since $\varphi$ is chosen arbitrarily from $C(X)$, we conclude that $x=x+t$ for all such $x$, contradicting the assumption that the flow has no fixed points.

(b) $\Rightarrow$ (a) If the flow had a fixed point $x$ then the point mass at $x$ would be invariant measure for the flow. The $L^{2}$ space of such a measure is $\mathbb{C}$. This contradicts (b).

\section{ANALYTIC REPRESENTATIONS OF THE FLOW}

Let $(\pi, P)$ be a nondegenerate analytic representation of the flow on a Hilbert space $K$. Define

$$
K_{+}=\bigvee\{\pi(\varphi) P K: \varphi \in C(X)\}
$$

and

$$
K_{-}=\bigcap_{\lambda>0}[\pi(C(X,[\lambda, \infty))) P K]^{-} .
$$

Obviously $K_{+}$is invariant for $\mathscr{A}$. Because of (1.2), (2.1), (2.3), and (2.4), $K_{-}$is also invariant for $\mathscr{A}$. Therefore, so is $K^{\prime}=K_{+} \ominus K_{-}$. Let $\mathscr{C} \mathscr{A}$ be the commutator ideal of $\mathscr{A}$. Then

$$
\mathscr{C} \mathscr{A}\left|\left(K \ominus K_{+}\right)=\{0\}=\mathscr{C} \mathscr{A}\right| K_{-} .
$$

Indeed, because both $K_{+}$and $K \ominus K_{+}$are invariant subspaces, for any $\varphi \in$ $C(X),[\pi(\varphi), P]\left(K \ominus K_{+}\right) \subset K_{+} \cap\left(K \ominus K_{+}\right)$. Hence $\mathscr{C} \mathscr{A} \mid\left(K \ominus K_{+}\right)=\{0\}$. On the other hand, it follows from (1.2) and (2.3) that $[\pi(\varphi), P] \mid[\pi(\psi) P K]^{-}=0$ if $\operatorname{sp}(\varphi) \subset[a, \infty)$ and $\operatorname{sp}(\psi) \subset(\max \{-a, 0\}, \infty)$. Therefore, $\mathscr{C} \mathscr{A} \mid K_{-}=\{0\}$. $K_{-}$and $K \ominus K_{+}$should be thought of as the "distant future" and "distant past" for the representation $(\pi, P)$.

Let $\pi^{\prime}$ be the restriction of $\pi$ of $K^{\prime}$; i.e., let $\pi^{\prime}(\varphi)=\pi(\varphi) \mid K^{\prime}$ for every $\varphi \in C(X)$. Define $P^{\prime}=\left(P-P_{-}\right) \mid K^{\prime}$, where $P_{-}$is the orthogonal projection onto $K_{-}$. Our analysis shows that $\left(\pi^{\prime}, P^{\prime}\right)$ is a nondegenerate analytic representation of the flow. Furthermore, the pair $\left(\pi^{\prime}, P^{\prime}\right)$ has the properties that

$$
\bigvee\left\{\pi^{\prime}(\varphi) P^{\prime} K^{\prime}: \varphi \in C(X)\right\}=K^{\prime}
$$

and

$$
\bigcap_{\lambda>0}\left[\pi^{\prime}(C(X,[\lambda, \infty))) P^{\prime} K^{\prime}\right]^{-}=\{0\} .
$$

In conformity with [9], which in turn was motivated in part by considerations from dilation theory, we call the restriction of $(\pi, P)$ to $K^{\prime}$ pure. Because of $(3.1), \mathscr{A} \mid\left(K \ominus K^{\prime}\right)$ is a commutative $C^{*}$-algebra. Thus every analytic representation of a flow decomposes as the direct sum of a pure representation and one whose image is abelian. Moreover, the pure summand is nonzero if and only if the representation is faithful. For our purpose here, only the pure part of $(\pi, P)$ plays a role. 
Suppose that the analytic representation $(\pi, P)$ is pure so that the equations

$$
\bigvee\{\pi(\varphi) P K: \varphi \in C(X)\}=K
$$

and

$$
\bigcap_{\lambda>0}[\pi(C(X,[\lambda, \infty))) P K]^{-}=\{0\}
$$

are satisfied. For each $\lambda \in \mathbb{R}$, let $P_{\lambda}$ denote the orthogonal projection from $K$ onto $[\pi(C(X,[\lambda, \infty))) P K]^{-}$. Obviously $P_{\lambda_{1}} \leq P_{\lambda_{2}}$ if $\lambda_{1}>\lambda_{2}$. It follows from (3.2) and (3.3) that $\lim _{\lambda \rightarrow-\infty} P_{\lambda}=1$ and $\lim _{\lambda \rightarrow+\infty} P_{\lambda}=0$. Thus

$$
E_{\lambda}=E((-\infty, \lambda])=1-\sup \left\{P_{s}: s>\lambda\right\}, \quad \lambda \in \mathbb{R},
$$

is a resolution of the identity on $K$. Since $1 \in C(X,[0, \infty))=A(X)$ and $\pi(A(X)) P=P \pi(A(X)) P$, we have $P=P_{0}$. Therefore, $P=P_{0} \geq$ $\sup \left\{P_{s}: s>0\right\}=1-E((-\infty, 0])=E((0, \infty))$. On the other hand, for any $\lambda<0, E((\lambda, \infty))=\sup \left\{P_{s}: s>\lambda\right\} \geq P_{0}=P$. Since $\inf \{E((\lambda, \infty)): \lambda<0\}=$ $E([0, \infty))$, we have

$$
E((0, \infty)) \leq P \leq E([0, \infty)) .
$$

It follows from (2.3) that $\pi(C(X,[\mu, \infty))) P_{\lambda} K \subset P_{\lambda+\mu} K$ for any $\lambda, \mu \in \mathbb{R}$. Therefore, for any $\lambda, \mu \in \mathbb{R}$ and $\varphi \in C(X,[\mu, \infty))$,

$$
\begin{aligned}
\pi(\varphi) E((\lambda, \infty)) K & =\pi(\varphi)\left[\bigvee\left\{P_{\gamma} K: \gamma>\lambda\right\}\right] \subset \bigvee\left\{P_{\gamma+\mu}: \gamma>\lambda\right\} \\
& =E((\lambda+\mu, \infty)) K
\end{aligned}
$$

and

$$
\begin{aligned}
\pi(\varphi) E([\lambda, \infty)) K & =\pi(\varphi)\left[\bigcap_{\gamma<\lambda} E((\gamma, \infty)) K\right] \subset \bigcap_{\gamma<\lambda} E((\gamma+\mu, \infty)) K \\
& =E([\lambda+\mu, \infty)) K .
\end{aligned}
$$

Let

$$
D=\int_{\mathbb{R}} \lambda d E_{\lambda}
$$

and let

$$
V(t)=\exp (i t D), \quad t \in \mathbb{R} .
$$

The following lemma is known as Forelli's Spectral-Commutation Theorem. It is a consequence of more general results whose proofs may be found in $[1,4$, 7, 10]. A direct, elementary proof is presented in [9].

Lemma 3.1. For every $\varphi \in C(X)$ and every $t \in \mathbb{R}, V(t) \pi(\varphi) V(-t)=\pi\left(\varphi_{t}\right)$, where $\varphi_{t}(x)=\varphi(x+t)$.

Let $\lambda_{0} \in \sigma(D)$, the spectrum of $D$, pick a sequence of unit vectors $y_{n} \in$ $E\left(\left(\lambda_{0}-1 / n, \lambda_{0}+1 / n\right)\right)$, let $L$ be a Banach generalized limit on $l^{\infty}\left(\mathbb{Z}_{+}\right)$, and define

$$
\rho(A)=L\left(\left\{\left\langle A y_{n}, y_{n}\right\rangle\right\}_{n=1}^{\infty}\right)
$$

for every bounded operator $A$ on $K$. Obviously $\rho$ is a state on $\mathscr{L}(K)$. 
Lemma 3.2. There exists an invariant probability measure $d m$ of the flow such that

for every $\varphi \in C(X)$.

$$
\rho(\pi(\varphi))=\int_{X} \varphi(x) d m(x)
$$

Proof. It suffices to show that the functional $\varphi \mapsto \rho(\pi(\varphi))$ is translation invariant, i.e., $\rho(\pi(\varphi))=\rho\left(\pi\left(\varphi_{t}\right)\right)$ for every $t \in \mathbb{R}$. By Lemma 3.1, we have

$$
\rho\left(\pi\left(\varphi_{t}\right)\right)=\rho(V(t) \pi(\varphi) V(-t))=L\left(\left\{\left\langle\pi(\varphi) V(-t) y_{n}, V(-t) y_{n}\right\rangle\right\}_{n=1}^{\infty}\right) .
$$

Because $y_{n} \in E\left(\left(\lambda_{0}-1 / n, \lambda_{0}+1 / n\right)\right) K$,

$$
\left\|V(-t) y_{n}-e^{-i \lambda_{0} t} y_{n}\right\| \leq \max _{|\lambda| \leq 1 / n}\left|e^{i \lambda t}-1\right| \rightarrow 0 \quad \text { as } n \rightarrow \infty .
$$

It follows from the properties of Banach generalized limits that

$$
\begin{aligned}
& L\left(\left\{\left\langle\pi(\varphi) V(-t) y_{n}, V(-t) y_{n}\right\rangle\right\}_{n=1}^{\infty}\right) \\
& \quad=L\left(\left\{\left\langle\pi(\varphi) e^{-i \lambda_{0} t} y_{n}, e^{-i \lambda_{0} t} y_{n}\right\rangle\right\}_{n=1}^{\infty}\right)=\rho(\pi(\varphi)) .
\end{aligned}
$$

Lemma 3.3. If the analytic representation $(\pi, P)$ of the flow is faithful, then there exists a $\varphi \in A(X)$ such that $P \pi(\varphi)(1-P) \pi(\bar{\varphi}) P \neq 0$.

Proof. We may, of course, assume that $(\pi, P)$ also satisfies (3.2) and (3.3). Let $d m$ be the measure provided by the previous lemma. Let $\delta=\int_{\mathbb{R}} \lambda d e_{\lambda}$ be the spectral decomposition of the infinitesimal generator of the unitary group $\left\{u_{t}: t \in \mathbb{R}\right\}$ on $L^{2}(X, d m)$. By Proposition 2.1, $\sigma(\delta) \cap\left(\left|\lambda_{0}\right|+3, \infty\right) \neq$ $\varnothing$. Therefore, by (2.5), there exist a $p>\left|\lambda_{0}\right|+3$ and a $\xi \in A(X)$ such that $\operatorname{sp}(\xi) \subset(p, p+1)$ and $\int_{X}|\xi|^{2} d m=1$. By (3.4) and (3.5), $\pi(\xi) y_{n} \in$ $E\left(\left(\left|\lambda_{0}\right|+3+\lambda_{0}-1, \infty\right)\right) K \subset E((2, \infty)) K \subset P K$. Hence for any $A \in \mathscr{L}(K)$,

$$
\rho(\pi(\bar{\xi}) P A P \pi(\xi))=\rho(\pi(\bar{\xi}) A \pi(\xi)) \text {. }
$$

By $(2.1)$ and $(2.3), \operatorname{sp}\left(\xi^{2}\right) \subset(2 p, \infty)$ and $\operatorname{sp}(\bar{\xi}) \subset(-p-1, \infty)$. Therefore, $\operatorname{sp}\left(\xi^{2} \bar{\xi}\right) \subset(p-1, \infty)$ and $\pi\left(\xi^{2} \bar{\xi}\right) E((-1, \infty)) K \subset E((p-2, \infty)) K \subset$ $E\left(\left(\lambda_{0}+1, \infty\right)\right) K$. Consequently $\pi\left(\bar{\xi}^{2} \xi\right) y_{n} \in E((-\infty,-1]) K \subset(1-P) K$. Hence

$$
(1-P) \pi\left(\bar{\xi}^{2} \xi\right) y_{n}=\pi\left(\bar{\xi}^{2} \xi\right) y_{n} .
$$

Combining (3.9), (3.10), and Lemma 3.2, we have

$$
\rho\left(\pi(\bar{\xi}) P \pi\left(\xi^{2}\right)(1-P) \pi\left(\bar{\xi}^{2}\right) P \pi(\xi)\right)=\rho\left(\pi\left(\bar{\xi} \xi^{2}\right) \pi\left(\bar{\xi}^{2} \xi\right)\right)=\int_{X}|\xi|^{6} d m .
$$

Because $\int_{X}|\xi|^{2} d m=1$, we have $\int_{X}|\xi|^{6} d m \neq 0$. Hence $P \pi\left(\xi^{2}\right)(1-P) \pi\left(\bar{\xi}^{2}\right) P$ $\neq 0$.

\section{THE PROOF OF THEOREM 1}

For each ideal $\mathscr{I} \subset \mathscr{T}$, let $\widehat{\mathscr{I}}$ denote the ideal in $\mathscr{A}$ generated by $\{A B C$ : $A, C \in \mathscr{A}, B \in \mathscr{I}\}$. Since $P \mathscr{A} P=\mathscr{T}$, we have $P \widehat{\mathscr{J} P}=\mathscr{I}$. It is obvious that $P \mathscr{C} \mathscr{A} P \subset \mathscr{C}_{1 / 2}$. On the other hand, because $P \pi(\varphi) P \pi(\psi) P-P \pi(\varphi \psi) P=$ $P([\pi(\varphi) P-P \pi(\varphi)] \pi(\psi)) P$ and $\pi(\varphi) P-P \pi(\varphi) \in \mathscr{C} \mathscr{A}$, we have $P \mathscr{C} \mathscr{A} P \supset \mathscr{C}_{1 / 2}$. Therefore $P \mathscr{C} \mathscr{A} P=\mathscr{C}_{1 / 2}$. Suppose now that $\mathscr{C}_{1 / 2} \neq \mathscr{C}$. Then, according to the 
above equality, $\hat{\mathscr{C}}$ would be a proper ideal in $\mathscr{C} \mathscr{A}$ and, consequently, $\mathscr{A} / \hat{\mathscr{C}}$ would be noncommutative. Let $\Phi: \mathscr{A} / \widehat{\mathscr{C}} \rightarrow \mathscr{L}(\widehat{K})$ be a faithful representation of $\mathscr{A} / \hat{\mathscr{C}}$ on a Hilbert space $\widehat{K}$. Because $\mathscr{A} / \widehat{\mathscr{C}}$ is noncommutative, the pair $\hat{\pi}(\varphi)=\Phi(\pi(\varphi)+\hat{\mathscr{C}})$ and $\widehat{P}=\Phi(P+\hat{\mathscr{C}})$ gives a nondegenerate analytic representation of the flow. According to Lemma 3.3, there exists a $\varphi \in A(X)$ such that $\widehat{P} \hat{\pi}(\varphi)(1-\widehat{P}) \hat{\pi}(\bar{\varphi}) \widehat{P} \neq 0$. On the other hand, since $\varphi \in A(X)$, we have

$$
P \pi(\varphi)(1-P) \pi(\bar{\varphi}) P=P \pi(\bar{\varphi}) P \pi(\varphi)-P \pi(\bar{\varphi}) P \pi(\varphi) P \in \mathscr{C} \subset \hat{\mathscr{C}} .
$$

Therefore

$$
\widehat{P} \hat{\pi}(\varphi)(1-\widehat{P}) \hat{\pi}(\bar{\varphi}) \widehat{P}=\Phi(P \pi(\varphi)(1-P) \pi(\bar{\varphi}) P+\widehat{\mathscr{C}})=0 .
$$

This contradiction proves the theorem.

\section{REFERENCES}

1. W. Arveson, On groups of automorphisms of operator algebras, J. Funct. Anal. 15 (1974), 217-243.

2. R. Curto, P. Muhly, and J. Xia, Toeplitz operators on flows, J. Funct. Anal. 93 (1990), 391-450.

3. R. Douglas, Another look at real-valued index theory (J. B. Conway and B. B. Morrel, eds.), Pitman Research Notes Math. Ser., vol. 192, Longman Sci. Tech., New York, 1988, pp. 91-120.

4. F. Forelli, Analytic and quasi-invariant measures, Acta Math. 118 (1967), 33-59.

5. I. Gohberg and N. Krupnik, The algebra generated by Toeplitz matrices, Funktsional Anal. i Prilozhen 3 (1969), 46-56; English transl., Funct. Anal. Appl. 3 (1969), 119-127.

6. R. Ji and J. Xia, On the classification of commutator ideals, J. Funct. Anal. 78 (1988), 208-232.

7. R. Loebl and P. Muhly, Analyticity and flows on von Neumann algebras, J. Funct. Anal. 29 (1978), 214-252.

8. P. Muhly, I. Putnam, and J. Xia, Contributions to the $K$-theory of $C^{*}$-algebras of Toeplitz operators and singular integral operator, Bull. Amer. Math. Soc. 21 (1989), 47-50.

9. __ On the $K$-theory of some $C^{*}$-algebras of Toeplitz and singular integral operators, J. Funct. Anal. (to appear).

10. G. Pedersen, $C^{*}$-algebras and their automorphism groups, Academic Press, London, New York, and San Francisco, 1979.

Department of Mathematics, The University of Iowa, Iowa City, Iowa 52242

E-mail address: pmuhly@vaxa.weeg.uiowa.edu

Department of Mathematics, State University of New York at Buffalo, Buffalo, NEW YORK 14214-3093 